\title{
Feature-level Data Fusion for Energy Consumption Analytics in Additive Manufacturing
}

\author{
Fu Hu, Ying Liu, Member, IEEE, Jian Qin, Xianfang Sun and Paul Witherell
}

\begin{abstract}
The issue of Additive Manufacturing (AM) energy consumption attracts attention in both industry and academia, as the increasing trend of AM technologies being employed in the manufacturing industry. It is crucial to analyze, understand, and manage the energy consumption of AM for better efficiency and sustainability. The energy consumption of AM systems is related to various correlated attributes in different phases of an AM process. Existing studies focus mainly on analyzing the impacts of different processing and material attributes, while factors related to design and working environment have not been paid enough attention to. Such factors involve features with various dimensions and nested structures that are difficult to handle in the analysis. To tackle these issues, a feature-level data fusion approach is proposed to integrate heterogeneous data in order to build an AM energy consumption model to uncover energy-relevant information and knowledge. A case study using real-world data collected from a selective laser sintering (SLS) system is presented to validate the proposed approach, and the results indicate that the fusion strategy achieves better performances on energy consumption prediction than the individual ones. Based on the analysis of feature importance, the geometry relevant features are found to have significant impacts on AM energy consumption.
\end{abstract}

\section{INTRODUCTION}

Additive manufacturing (AM), also referred to as rapid prototyping [1], creates physical objects from a geometrical representation by successive addition of materials [2]. Due to its unique production paradigm, AM technologies have been increasingly used for mass customization, production of any types of open-source designs in the field of agriculture, healthcare, automotive industry, and aerospace industries [3]. Comparing to conventional manufacturing, AM shows higher efficiency and flexibility, leading to its increasing adoption in the industry. However, the Life Cycle Analysis (LCA) indicates that the energy consumption of AM systems tends to have a significant effect on the environment [4]. This drives AM energy consumption analytics to a crucial research topic of AM sustainability as the number of AM systems being employed keeps growing. AM is regarded as a complex manufacturing system of which the energy consumption can be different due to various AM technologies, such as selective laser sintering (SLS), Stereolithography (SLA), and electron beam melting (EBM). Hence, discovering and understanding the relationship between AM processes and energy consumption can significantly contribute to the improvement of energy management.

$\mathrm{Fu} \mathrm{Hu}$ and Ying Liu are with the Institute of Mechanical and Manufacturing Engineering, School of Engineering, Cardiff University, Cardiff, UK(corresponding author e-mail: huf4@ cardiff.ac.uk).

Jian Qin is with the Welding Engineering and Laser Processing Centre, School of Aerospace, Transport and Manufacturing, Cranfield University, Cranfield, UK.
The energy consumption is correlated with various subsystems and factors, showing a large difference in terms of different working principles and main material supplies [5-8]. Most researchers have been focusing on investigations of the relationships between processing attributes, material properties, and energy consumption, while the impacts of design-relevant and working environmental attributes have not been paid enough attention to. Several factors related to design models and working environment, such as the volume of part envelopes [8], part geometry [9], platform temperature [10], have been identified to have strong relationships with energy consumption in AM processes. However, to comprehensively analyze and understand the impacts of these correlated factors is still challenging. With the advancement of data collection and storage technologies, data-driven approaches have been increasingly adopted to investigate and discover the hidden knowledge in digital manufacturing systems such as AM. In general, data is generated from different sources and subsystems of AM processes, such as building chambers, operation, and control systems. This data is rarely independent but nested to capture the complexity of various factors that are correlated with energy consumption. Hence, it is crucial to jointly analyze these correlated factors for improving the energy management of AM systems.

This paper proposed a feature-level data fusion approach for AM energy consumption analytics through design-relevant and working environment data. Section II reviews the studies of analysis for AM energy consumption and the applications of data fusion techniques in manufacturing. In Section III, a feature-level data fusion approach is proposed to integrate the features extracted from design models and working environment data for energy consumption prediction. Section IV presents a case study of energy consumption prediction and analytics on an SLS system. Results are compared and discussed the performance of the proposed approach. The impacts of features are analyzed based on the predictive model and the statistical correlation coefficient. In Section V, the benefits and restrictions of the proposed methods are concluded.

\section{LITERATURE REVIEW}

\section{A. Energy Consumption Analysis for AM Systems}

The energy consumption of AM systems is affected by different attributes. Different researches that focus on various energy consumption relevant features in AM processes are

Xianfang Sun is with the School of Computer Science and Informatics, Cardiff University, Queen's Buildings, Cardiff CF24 3AA, UK.

Paul Witherell is with the National Institute of Standards and Technology, 100 Bureau Drive, Gaithersburg, MD 20899, USA. 
shown in Table I. These features can be classified into four categories in terms of product design, processing, working environment, and material. However, the impacts of these attributes on AM energy consumption are inconsistent which is largely due to different working principles of AM systems and inadequate factors that were explored. Thus, it is hard to identify and measure all the correlated factors in AM processes from a single study and experiment. For instance, processing attributes have been investigated in several studies. Sreenivasan and Bourell [7] calculated the energy consumption of the target SLS system by adopting a basic function in which the main inputs are the average current flowing and line voltage. Their results showed that the chamber heater was the largest energy consumer and scan speed, laser power rate, road width size, and material density were also correlated with energy consumption in the target SLS system. Ma et al. [11] developed a multi-objective optimization of process attributes model considering energy consumption and material cost. In this study, scan speed, gap distance, and layer thickness were taken into account and found to have significant impacts on energy consumption in the target AM system. Besides processing attributes, Watson and Taminger [8] also investigated the impacts of designrelevant attributes, such as the volume of deposited material and volume defined by the part envelope, on AM energy consumption. However, their suggested computational energy consumption model was not validated by experiments. A mathematical model for AM energy consumption of SLAbased processes was proposed by Yang et al. [12]. The author calculated total energy consumption of the target system from three subsystems which were categorized as ultraviolet curing process, building platform movement, and cooling system. The impacts of orientation, layer thickness, the curing time for stable layers and transition rate on energy consumption were analyzed.

Besides the processing and material attributes, a few design-relevant and working environmental features have been investigated in previous studies. Liu et al. [9] analyzed AM energy consumption from machine level and process level, suggesting that energy consumption can be reduced by improving the capacity utilization to dilute the energy consumed in the preheating and cooling down process. Moreover, in situ temperature monitoring is considered critical for understanding the heat loss and energy flow during the AM process by the author. This reveals that the attributes of the working environment, e.g., chamber temperature and gas flow, are closely correlated with the thermal history of the substrate and AM energy consumption. In the scope of big data and IoT applications, Qin et al. [10] proposed a data analytics model for predicting AM energy consumption based on artificial neural networks. The prediction model integrates data from design-relevant, process operation, working environment, and material, which tended to cover the entire data generation stages during an AM process. The results showed that the design-relevant and working environment attributes had significant impacts on AM energy consumption based on the weights of neurons. However, the relationship between features and energy consumption was not analyzed, of which further investigation is needed.

According to previous studies, researchers mainly focused on investigating the impacts of processing and material attributes on AM energy consumption, while the impacts of product design and working environment attributes have not been fully explored. Hence, it is crucial to analyze and understand the factors related to the design and working environment. In the next section, the applications of data fusion techniques in manufacturing are discussed.

TABLE I. ENERGY CONSUMPTION RELATED ATTRIBUTES OF AM SYSTEMS IN LITERATURE

\begin{tabular}{|c|c|c|c|c|}
\hline $\begin{array}{l}\text { Previous } \\
\text { Studies }\end{array}$ & $\begin{array}{c}\text { Product } \\
\text { design }\end{array}$ & Processing & $\begin{array}{c}\text { Working } \\
\text { environme } \\
\text { nt }\end{array}$ & Material \\
\hline $\begin{array}{l}\text { Bourell } \\
\text { et al. [7] }\end{array}$ & N/A & $\begin{array}{l}\text { Scan speed; } \\
\text { Laser power } \\
\text { rate; Road } \\
\text { width size }\end{array}$ & N/A & $\begin{array}{l}\text { Material } \\
\text { density }\end{array}$ \\
\hline $\begin{array}{l}\text { Telenko } \\
\text { et al. [6] }\end{array}$ & $\begin{array}{l}\text { Build } \\
\text { height }\end{array}$ & N/A & N/A & $\begin{array}{l}\text { Material } \\
\text { density }\end{array}$ \\
\hline $\begin{array}{l}\text { Watson } \\
\text { et al. [8] }\end{array}$ & $\begin{array}{l}\text { Deposited } \\
\text { material } \\
\text { volume; } \\
\text { Part } \\
\text { envelope } \\
\text { volume. }\end{array}$ & $\begin{array}{l}\text { Transported } \\
\text { distance of } \\
\text { feedstock and } \\
\text { recycling; Build } \\
\text { platform size }\end{array}$ & N/A & N/A \\
\hline $\begin{array}{l}\text { Ma et al. } \\
\text { [11] }\end{array}$ & N/A & $\begin{array}{l}\text { Scan speed; } \\
\text { Gap distance; } \\
\text { Layer thickness }\end{array}$ & N/A & N/A \\
\hline $\begin{array}{l}\text { Yang et } \\
\text { al. [12] }\end{array}$ & $\begin{array}{l}\text { Orientatio } \\
-n\end{array}$ & $\begin{array}{l}\text { Layer thickness; } \\
\text { Curing time for } \\
\text { stable layers; } \\
\text { Curing time } \\
\text { transition rate }\end{array}$ & N/A & N/A \\
\hline $\begin{array}{l}\text { Liu et } \\
\text { al. [9] }\end{array}$ & $\begin{array}{l}\text { Part } \\
\text { geometry }\end{array}$ & $\begin{array}{l}\text { Laser power; } \\
\text { Pre-heating; } \\
\text { Scanning speed }\end{array}$ & $\begin{array}{l}\text { In situ } \\
\text { temperature }\end{array}$ & $\begin{array}{l}\text { Material } \\
\text { type }\end{array}$ \\
\hline $\begin{array}{l}\text { Qin et } \\
\text { al. [10] }\end{array}$ & $\begin{array}{l}\text { Part } \\
\text { geometry } \\
\text { Build } \\
\text { height } \\
\text { Filling } \\
\text { degree }\end{array}$ & $\begin{array}{l}\text { Dispenser; } \\
\text { Recoater speed; } \\
\text { Hatch power; } \\
\text { Hatch speed; } \\
\text { Hatch width; } \\
\text { Hatch angle }\end{array}$ & $\begin{array}{l}\text { Lab } \\
\text { temperature } \\
\text {; Frame } \\
\text { temperature } \\
\text {; Platform } \\
\text { temperature } \\
; \mathrm{O}_{2} \text { level; }\end{array}$ & $\begin{array}{l}\text { Material } \\
\text { temperat- } \\
\text { ure } \\
\text { and } \\
\text { humidity }\end{array}$ \\
\hline
\end{tabular}

\section{B. Data Fusion in Manufacturing}

The data fusion is defined as a framework [13], fit by an ensemble of tools, for the joint analysis of data from multiple sources or modalities to uncover information not recoverable by the individual ones. In general, data fusion techniques are commonly categorized into three levels: data-level, featurelevel, and decision-level [14]. Data-level fusion is directly combining and integrating data collected from multiple sources to build a model, while features are required to be extracted from raw data for integration in feature-level fusion. Decision-level fusion is to combine the output of several models into one model for improving decisions. Initially, data fusion techniques are used to fuse data from multiple sensors and related information from the associated database since it provides significant advantages over single-source data. With the fast development of IoT and advanced data analytics techniques, it is increasingly used for studying complex systems in the industry, especially tackling issues and challenges in manufacturing systems.

A feature-level data fusion approach was proposed by $\mathrm{Wu}$ et al. [15] for predicting surface roughness in fused deposition modeling (FDM) processes. In this paper, features were extracted from the real-time monitoring data collected from multi-sensors, including temperature and vibration of the build 
plate, the temperature and vibration of the extruder, and the temperature of the deposited material. These features were fused as input for building the predictive model of surface roughness and showed high accuracy. The author also considered that using feature-level fusion for modeling is better than data-level fusion in terms of computational efficiency. However, only signal data collected from multiple sensors were fused for modeling by the author, and similar methods of fusion are explored in these studies [16-18].

When dealing with massive, correlated, and multidimensional data, data fusion techniques show their advantages in discovering hidden knowledge and information by jointly analyzing. Vandone et al. [19] developed a comprehensive data fusion approach, extracting features from sensor signals, image data, 3D geometry, and machine files, to model AM process and provide process control strategy. However, only laser power and tool velocity were selected as process parameters and the control strategy was not validated by experiments. Cai et al. [20] proposed a framework of fusing manufacturing data and sensory data into developing "digitaltwins" virtual machine tools based on the data fusion approach, which aimed to improve the accountability and capabilities for cyber-physical manufacturing. The characteristics profile of a machine tool was constructed by the key features extracted from the manufacturing process and sensory data, such as feed rate, spindle current, and acceleration. However, only one milling machine tool and a limited number of machining sensory data were implemented in this paper, further work on comprehensively formatting characteristics profiles of the virtual machine tool by fusing more extracted features is needed. A hybrid approach was proposed by Zhang et al. [21] to fuse image data collected from cameras and sensor data for identifying items in smart refrigerators. Besides features-level fusion, the author also adopted multi-model fusion based on neural networks to improve the accuracy of recognition. The results showed that the accuracy of the fused model was $5 \%$ higher than the individual model.

According to previous studies, researchers benefit from using data fusion techniques for modeling or analyzing complex processes and systems. Multiple sensors and IoT applications enable researchers to acquire more information and data generated from the production chain in manufacturing systems. However, as the collected data and information become more diverse, massive, and irregular, it is difficult to simply combine and fuse data especially when the data is nested and hierarchical. For AM systems, data is generated with different dimensions and structures, building up barriers to conventional data analysis methods. Thus, it is significant to fuse the data sources and integrate the collected data. In the next section, a feature-level fusion based analytics model is proposed, which focuses on analyzing the correlated features on AM energy consumption.

\section{METHODOLOGY}

\section{A. Data Sensing and Collection}

The data used for analyzing the relationship between the design-relevant, the working environment, and the energy consumption are primarily collected from the design models, the systems monitoring files, and different sensors. This collected data are generated from the AM process during different builds. The design-relevant data is collected from design models and analyzed by using AM software where the information of features such as the geometries, part locations, and orientations can be obtained. The machine embedded sensors and IoT applications are used for collecting working environment data automatically [22]. Information on temperature, humidity, and gas concentration, etc., can be obtained. After data collection, feature extraction and selection techniques are applied before fusion into the predictive model. Then, the features are analyzed based on the feature importance ranking. Statistical correlation coefficient analysis is a prevailing method in measuring the linear correlation between variables. This method is adopted in this study to measure the strength of the associations between the factors and energy consumption from the statistical perspective. The framework of the proposed methodology is illustrated in Figure 1.

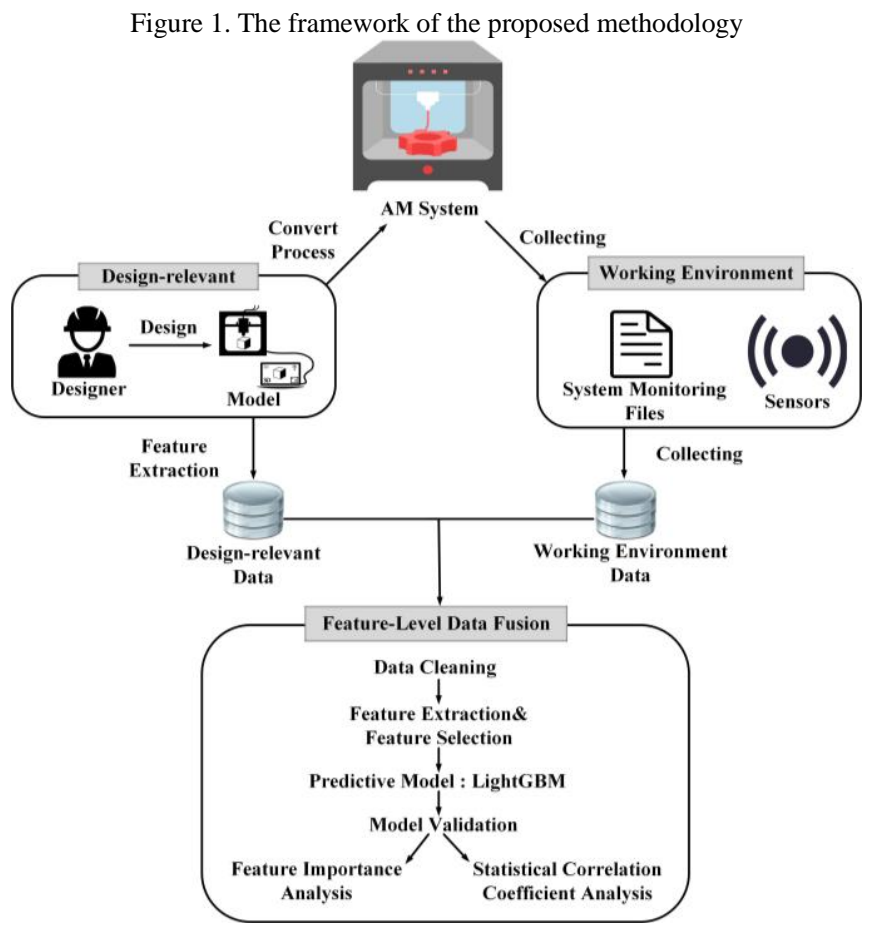

\section{B. Feature-level Data Fusion}

AM energy consumption is correlated with various attributes and factors. It is difficult to analyze and understand these factors separately. Data fusion techniques provide effective ways to uncover hidden knowledge through joint analysis. During an AM process, the data is generated with two different levels, layer-level and build-level. As the data remains the same during an AM process and is collected once in a build, the design-relevant data is classified into the buildlevel. For the working environment, the data collected from sensors keeps changing all the time as the parts are manufactured layer by layer. Therefore, the working environment data is usually collected once per layer and should be classified as layer-level data.

Considering that the types and levels of this generated data are various, it is difficult to fuse the raw data for modeling and analyzing directly. Hence, a feature-level data fusion method is adopted to fuse these data sources. It is effective to extract features from raw data which aims to reduce data 
dimensions without losing significant information. Additionally, the raw data collected from sensors normally has massive volume and contains noise data. The predictive model based on feature-level fusion is more computationally efficient as it processes extracted features that are more informative than raw data. In the build-level, the design-relevant features are analyzed and extracted by using AM software. In the layerlevel, different time-domain features are extracted from working environment data. In this study, time-domain features in Ref. [23] are considered, including standard deviation, minimum, maximum, mean, and amplitude values. After features are extracted from the raw data, a feature selection process is employed to select a subset of features from the input features. Feature selection could largely reduce negative impacts from noise or irrelevant features [24]. Moreover, these selected features with crucial information can effectively describe the input features and improve the prediction performance.

Following this, the time-domain features related to the working environment are extracted from layer-level data and can be fused with design-relevant features at build-level for modeling and analyzing.

\section{Predictive Model for Energy Consumption Analytics}

Machine learning technologies and data fusion strategies have demonstrated their capabilities of finding patterns in data and applying this knowledge in industrial scenarios. Hence, a machine learning method has been developed to model this nonlinear system, providing insights into the highly complicated relationships. The design-relevant features and the time-domain features extracted from the working environment data are integrated and fed into the machine learning model for analysis. The output of the model is the unit energy consumption which is described in section $\mathrm{D}$.

To tackle with massive data and obtain relatively high accuracy of predictions, a predictive model for AM energy consumption was developed based on Light Gradient Boosting Machine (LightGBM) [25]. LightGBM is an open-source promotion framework based on the Gradient Boosting Decision Tree (GBDT) algorithm that strengthens weak learners into a strong one and mainly tackles classification and prediction tasks. For GBDT algorithm, when trains a dataset $\left\{\left(x_{1}, y_{1}\right),\left(x_{2}, y_{2}\right), \ldots,\left(x_{\mathrm{n}}, y_{\mathrm{n}}\right)\right\}$, where $x$ represents data instances and $y$ represents the target to be predicted and the estimated function is represented by $F(x)$, the optimization goal of GBDT is to minimize the loss function $L(y, F(x))$ :

$$
F=\underset{F}{\arg \min } E_{x y}[L(y, F(x))]
$$

The loss function is minimized by iteration in line search:

$$
F_{m}(x)=F_{m-1}(x)+\gamma_{m} h_{m}(x)
$$

where $h_{m}(x)$ represents the base decision tree, $m$ is the iteration number, and $\gamma_{m}=\arg \min _{\gamma} \sum_{i=1}^{n} L\left(y_{i}, F_{m-1}\left(x_{i}\right)+\gamma h_{m}\left(x_{i}\right)\right)$.

LightGBM adopts a histogram-based decision tree algorithm and leaf-wise leaf growth strategy with depth limitation, largely reducing the storage and computational costs and ensuring high accuracy at the same time. The split points are determined by calculating variance gain in LightGBM which adopts gradient-based one-side sampling
(GOSS). Additionally, to tackle sparse features, LightGBM employs exclusive feature bundling (EFB) to avoid additional computational and memory overhead by converting features to a multi-dimensional one-hot feature. As LightGBM is an ensemble model of decision trees, the final model $F_{M}(x)$ can be obtained through a weighted combination.

$$
F_{M}(x)=\sum_{m=1}^{M} \gamma_{m} h_{m}(x)
$$

The model developed in this paper integrates the features extracted from design-relevant and working environment data for predicting AM energy consumption.

\section{Validation of the Predictive Model}

It is known that the energy consumption of an AM process largely depends on the building time for manufacturing parts. In other words, for the same process, the longer the building time is, the more energy is consumed. Hence, the energy consumption level is evaluated by using the unit energy consumption $E_{U}(\mathrm{kWh} / \mathrm{kg})$ of each printed build in this study.

$$
E_{U}=\frac{E_{B}}{M_{B}}
$$

In equation (4), $E_{B}$ represents the energy consumption of a total build, and $M_{B}$ is the weight of a total build. Model Correlation Coefficient (MCC) [26] is adopted to evaluate the accuracy of the prediction. MCC is calculated as:

$$
\begin{gathered}
M C C=\frac{S_{P A}}{\sqrt{S_{P} S_{A}}} \\
S_{P A}=\frac{\sum_{i}\left(P_{i}-\bar{P}\right)\left(a_{i}-\bar{a}\right)}{n-1} ; S_{P}=\frac{\sum_{i}\left(P_{i}-\bar{P}\right)^{2}}{n-1} ; S_{A}=\frac{\sum_{i}\left(a_{i}-\bar{a}\right)^{2}}{n-1} .
\end{gathered}
$$

In equation (5) and (6), $a_{i}$ is the actual data value and $\bar{a}$ is the average value of the whole data, $p_{i}$ is the predicted value of data, $\bar{p}$ is the average value of predicted data. The 5-fold cross-validation is used for evaluating the MCC performance.

\section{CASE STUdY}

In this case study, the data was collected from August 2016 to October 2019 from an SLS machine (EOS P700). Data and information of more than a hundred production processes with thousands of produced parts were included in the datasets. The parts were designed by different designers with various shapes and geometries.

\section{A. Data Pre-processing}

The design-relevant and working environment data was collected from each build in the target AM system. The designrelevant data was collected from CAD models which contain significant information about product design. Design-relevant features, such as the number of printed parts (NumPart), the height of the printed build (BuildHeight), the ratios between length, width, and height of the printed parts and builds, were extracted from CAD models using the AM analysis software, called Autodesk Netfabb. There were 12 design features that were extracted for describing the geometric information and spatial proportion information. For example, the filling degrees of the printed parts and builds were calculated for describing the utilization of the build chamber space. The working environment data was automatically collected once 
per layer by various system-embedded sensors. Attributes such as chamber temperature, frame temperature, platform temperature, and oxygen level are included in the data file where there are 9 attributes in total. The time-domain features were extracted from the working environment data and 45 features were finally obtained. A variance thresholding feature selection process was adopted to remove features that rarely changed in the datasets and the 21 features were finally selected.

\section{B. Results and Discussion}

In this case study, the results focused on the validation of the proposed data fusion approach and analyzing the impacts of product design and working environment on AM energy consumption. Support Vector Regression (SVR), $k$-Nearest Neighbors $(k-\mathrm{NN})$, and Random Forests (RFs) are introduced as benchmarks for comparison and to verify the performances of the proposed fusion method. The importance of each feature contributing to energy consumption was calculated and analyzed. Besides, the statistical correlation coefficient was adopted to describe the relationship between features and energy consumption.

The MCC results of LightGBM, SVR, $k$-NN, and RFs are shown in Figure 2. The performances of using three different datasets, i.e., the design-relevant, the working environment, and the combination of the entire datasets, for predicting energy consumption are presented. In general, the best MCC result was obtained respectively in four models by fusing the design-relevant and working environment data sources as input, while the lowest results are obtained by only using the working environment dataset as input. Specifically, the best result $(0.65)$ was obtained by the proposed LightGBM model and is slightly higher than the result (0.63) of the RFs model.

TABLE II. DESCRIPTION OF THE TOP 10 MOST IMPORTANT FEATURES IN FEATURE IMPORTANCE RANKING

\begin{tabular}{|l|l|}
\hline \multicolumn{1}{|c|}{ Feature } & \multicolumn{1}{c|}{ Description } \\
\hline FillingDegBuild & The filling degree of the whole printed build (\%) \\
\hline $\begin{array}{l}\text { AverFilling } \\
\text { DegPart }\end{array}$ & $\begin{array}{l}\text { The average filling degree of the single printed part } \\
(\%)\end{array}$ \\
\hline $\begin{array}{l}\text { AverRatio } \\
\text { L-WBuild }\end{array}$ & $\begin{array}{l}\text { The ratio of length to width of the whole printed build } \\
(\%)\end{array}$ \\
\hline AverPartHeight & The average height of the printed parts (mm) \\
\hline $\begin{array}{l}\text { AverRatio } \\
\text { L-HPart }\end{array}$ & $\begin{array}{l}\text { The average ratio of length to height of the single } \\
\text { printed part (\%) }\end{array}$ \\
\hline $\begin{array}{l}\text { Chamber } \\
\text { TemSD }\end{array}$ & $\begin{array}{l}\text { The standard deviation of the temperature value of } \\
\text { the building chamber }\left({ }^{\circ} \mathrm{C}\right)\end{array}$ \\
\hline O2LevelMax & $\begin{array}{l}\text { The maximum value of oxygen percentage in the } \\
\text { working chamber during a build }(\%)\end{array}$ \\
\hline $\begin{array}{l}\text { AverRatioL- } \\
\text { WPart }\end{array}$ & $\begin{array}{l}\text { The average ratio of length to width of the single } \\
\text { printed part }(\%)\end{array}$ \\
\hline BottomArea & The area of the bottom (mm $\left.{ }^{2}\right)$ \\
\hline NumPart & The number of printed parts \\
\hline
\end{tabular}

The ranking of features is obtained based on the proposed LightGBM model by calculating the average information gain in 5 predictions and processed with normalization, illustrated in Figure 3. The features rank in the top 10 of the feature importance ranking are described in Table II. In general, the entire impact of design-relevant features (75\%) is significantly larger than that of working environment features (25\%), which indicates that product design is strongly correlated with the unit energy consumption of the AM system. Specifically, the three most important features are FillingDegBuild, AverFillingDegPart, and AverRatioL-WBuild. The filling degree of the whole printed build has a strong impact on energy consumption (over 35\%), indicating that the utilization of the build chamber space is crucial. Besides, the changes in the temperature of the chamber also strongly correlated with energy consumption which possibly due to the heating process and heat transfer with materials. It is interesting that the height of the printed build and the number of printed parts appear not to have significant impacts on unit energy consumption which can be caused by various factors, such as different geometries, different filling degrees, and so on.

Figure 2. Comparison of MCC of the proposed model and benchmarks.

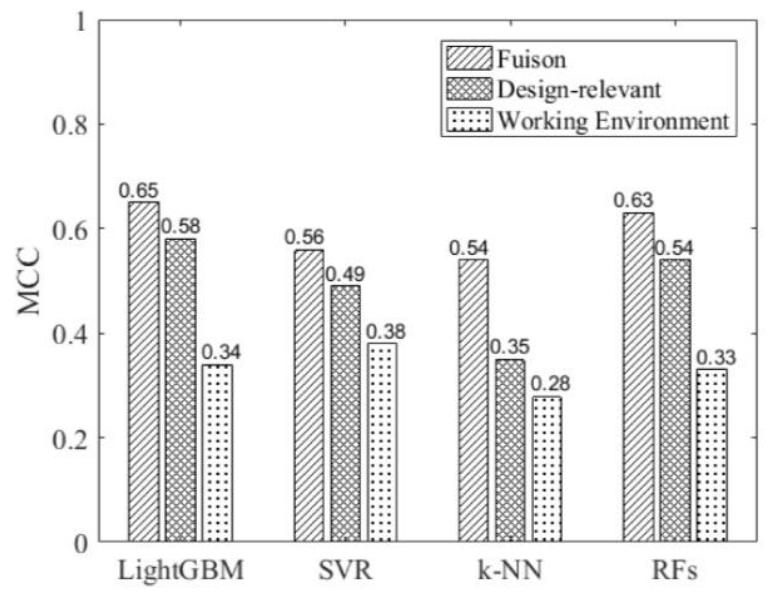

Figure 3. The feature importance ranking

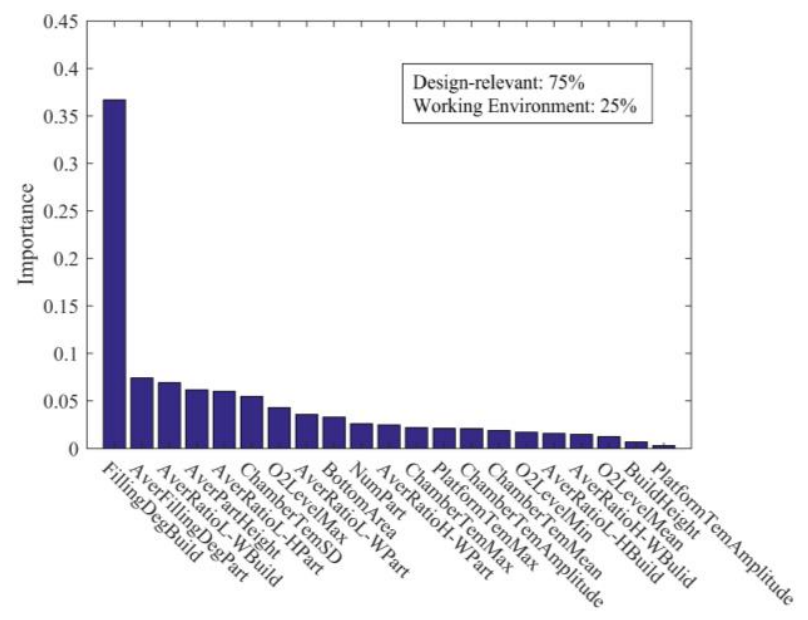

The features rank in the top 10 of the feature importance ranking are selected for correlation coefficient analysis, illustrated in Figure 4. In general, the FillingDegBuild and AverFillingDegPart show strong linear correlations (-0.57 and -0.28) with energy consumption in the target SLS system, which is consistent with the results of the feature importance analysis. Specifically, except for the AverRatioL-WBuild, AverRatioL-WPart, O2LevelMax, and AverRatioL-WPart, the remaining features show negative linear correlations with energy consumption. For instance, energy consumption tends to decrease when FillingDegBuild and BottomArea increase. The number of printed parts shows a negative correlation with 
energy consumption which is possibly due to the increase of the utilization of build chamber space. The energy consumption tends to increase while the temperature of the chamber with less fluctuation. Further exploration and investigation are needed to explain this phenomenon.

Figure 4. Statistical correlation coefficient analysis for the top 10 most important features

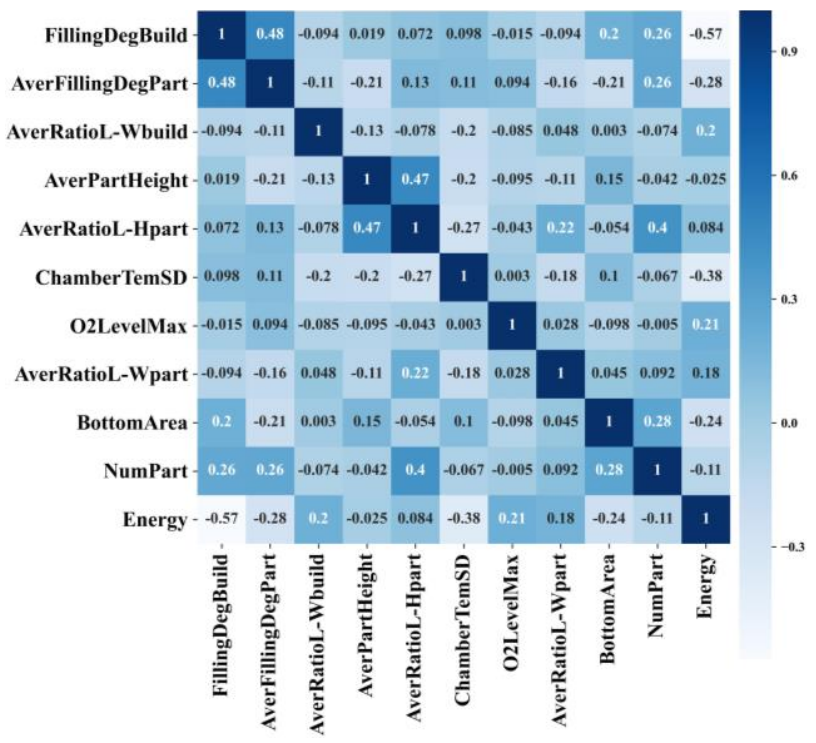

\section{CONCLUSIONS}

In this paper, a feature-level data fusion approach is developed for analyzing the features related to the design and working environment on AM energy consumption. The LightGBM model adopted in this paper can effectively explain the importance of features based on the information gain. An experimental study on an SLS system was implemented and the results indicated that better performances of prediction were obtained by integrating features as input in the predictive models. The feature importance analysis illustrated that the filling degree of the whole printed build had the highest impact (over to 35\%) on AM energy consumption among 21 selected features. In addition, the results also indicated that the entire impacts of design-relevant features (more than 70\%) on AM energy consumption were significant.

In future work, more design-relevant features will be extracted and factors such as material and processing attributes will also be taken into consideration for uncovering energy-relevant information and improving prediction accuracy. Energy consumption of different industrial AM systems such as SLM will be investigated in the future.

\section{REFERENCES}

[1] J.-P. Kruth, M.-C. Leu, T.J.C.A. Nakagawa, Progress in additive manufacturing and rapid prototyping, Cirp Annals, 47 (1998) 525-540.

[2] ISO/PRF, "Additive manufacturing -- General principles -- Part 1: Terminology", 2015.

[3] Ö. Keleş, C.W. Blevins, K.J.J.R.P.J. Bowman, Effect of build orientation on the mechanical reliability of 3D printed ABS, Rapid Prototyping Journal, 23 (2017) 320-328.
[4] R. Huang, M. Riddle, D. Graziano, J. Warren, S. Das, S. Nimbalkar, J. Cresko, E.J.J.o.C.P. Masanet, Energy and emissions saving potential of additive manufacturing: the case of lightweight aircraft components, Journal of Cleaner Production, 135 (2016) 1559-1570.

[5] B.C. Gross, J.L. Erkal, S.Y. Lockwood, C. Chen, D.M. Spence, Evaluation of 3D printing and its potential impact on biotechnology and the chemical sciences, ACS Publications, 2014.

[6] C. Telenko, C.C. Seepersad, Assessing energy requirements and material flows of selective laser sintering of Nylon parts, Proceedings of the Solid Freeform Fabrication Symposium, 2010, pp. 8-10.08.

[7] R. Sreenivasan, D. Bourell, Sustainability Study in Selective Laser Sintering- An Energy Perspective, Minerals, Metals and Materials Society/AIME, 420 Commonwealth Dr., P. O. Box ..., 2010.

[8] J. Watson, K.J.J.o.C.P. Taminger, A decision-support model for selecting additive manufacturing versus subtractive manufacturing based on energy consumption, Journal of Cleaner Production, 176 (2018) 1316-1322.

[9] Z. Liu, C. Li, X. Fang, Y.J.P.M. Guo, Energy consumption in additive manufacturing of metal parts, Procedia Manufacturing, 26 (2018) 834845.

[10] J. Qin, Y. Liu, R. Grosvenor, F. Lacan, Z.J.J.o.C.P. Jiang, Deep learning-driven particle swarm optimisation for additive manufacturing energy optimisation, Journal of Cleaner Production, 245 (2020) 118702.

[11] F. Ma, H. Zhang, K. Hon, Q.J.J.o.C.P. Gong, An optimization approach of selective laser sintering considering energy consumption and material cost, Journal of Cleaner Production, 199 (2018) 529-537.

[12] Y. Yang, L. Li, Y. Pan, Z.J.J.o.I.E. Sun, Energy consumption modeling of stereolithography - based additive manufacturing toward environmental sustainability, Journal of Industrial Ecology, 21 (2017) S168-S178.

[13] M. Cocchi, Data Fusion Methodology and Applications, Elsevier2019.

[14] D.L. Hall, J.J.P.o.t.I. Llinas, An introduction to multisensor data fusion, Proceedings of the IEEE, 85 (1997) 6-23.

[15] D. Wu, Y. Wei, J.J.I.J.o.P.R. Terpenny, Predictive modelling of surface roughness in fused deposition modelling using data fusion, International Journal of Production Research, 57 (2019) 3992-4006.

[16] J. Yuqin, W. Peixia, L. Yue, Study of manufacturing system based on neural network multi-sensor data fusion and its application, IEEE International Conference on Robotics, Intelligent Systems and Signal Processing, 2003. Proceedings. 2003, IEEE, 2003, pp. 1022-1026.

[17] J.A. Duro, J.A. Padget, C.R. Bowen, H.A. Kim, A.J.M.s. Nassehi, s. processing, Multi-sensor data fusion framework for CNC machining monitoring, Mechanical systems signal processing, 66 (2016) 505-520.

[18] Z. Liu, W. Guo, Z. Tang, Y.J.S. Chen, Multi-sensor data fusion using a relevance vector machine based on an ant colony for gearbox fault detection, Sensors, 15 (2015) 21857-21875.

[19] A. Vandone, S. Baraldo, A.J.I.R. Valente, A. Letters, Multisensor data fusion for additive manufacturing process control, IEEE Robotics Automation Letters, 3 (2018) 3279-3284.

[20] Y. Cai, B. Starly, P. Cohen, Y.-S.J.P.M. Lee, Sensor data and information fusion to construct digital-twins virtual machine tools for cyber-physical manufacturing, Procedia Manufacturing, 10 (2017) 1031-1042.

[21] W. Zhang, Y. Zhang, J. Zhai, D. Zhao, L. Xu, J. Zhou, Z. Li, S.J.C.i.I. Yang, Multi-source data fusion using deep learning for smart refrigerators, Computers in Industry, 95 (2018) 15-21.

[22] J. Qin, Y. Liu, R.J.A.E.I. Grosvenor, Multi-source data analytics for AM energy consumption prediction, Advanced Engineering Informatics, 38 (2018) 840-850.

[23] M. Muzammal, R. Talat, A.H. Sodhro, S.J.I.F. Pirbhulal, A multisensor data fusion enabled ensemble approach for medical data from body sensor networks, Information Fusion, 53 (2020) 155-164.

[24] R. Zhang, F. Nie, X. Li, X.J.I.F. Wei, Feature selection with multi-view data: A survey, Information Fusion, 50 (2019) 158-167.

[25] G. Ke, Q. Meng, T. Finley, T. Wang, W. Chen, W. Ma, Q. Ye, T.-Y. Liu, Lightgbm: A highly efficient gradient boosting decision tree, Advances in neural information processing systems, 2017, pp. 31463154.

[26] J. Han, J. Pei, M. Kamber, Data mining: concepts and techniques, Elsevier2011. 